Research Paper

\title{
Apolipoprotein A-I Is a Prognosticator of Nasopharyngeal Carcinoma in the Era of Intensity- modulated Radiotherapy
}

\author{
Hui Chang*, Jia-wang Wei*, Kai Chen, Shu Zhang, Fei Han, Li-xia Lu, Wei-wei Xiao ${ }^{凶}$, Yuan-hong Gao ${ }^{凶}$ \\ Department of Radiation Oncology, Sun Yat-sen University Cancer Center; State Key Laboratory of Oncology in South China; Collaborative Innovation Center \\ of Cancer Medicine; Guangzhou, Guangdong 510060, China. \\ * contributed equally to this work. \\ $\triangle$ Corresponding authors: Prof. Wei-wei Xiao and Prof. Yuan-hong Gao. Department of Radiation Oncology, Sun Yat-sen University Cancer Center. 651 \\ Dongfeng Road East, Guangzhou, Guangdong 510060, China. Prof. Wei-wei Xiao: Tel: +86-13710390520, E-mail: xiaoww@sysucc.org.cn. Prof. Yuan-hong Gao: \\ Tel: +86-13560182168, Fax: +86-020-87343491, E-mail: gaoyh@sysucc.org.cn. \\ (C) Ivyspring International Publisher. This is an open access article distributed under the terms of the Creative Commons Attribution (CC BY-NC) license \\ (https://creativecommons.org/licenses/by-nc/4.0/). See http://ivyspring.com/terms for full terms and conditions.
}

Received: 2017.09.15; Accepted: 2017.12.08; Published: 2018.01.11

\begin{abstract}
Background: In the era of intensity-modulated radiotherapy (IMRT), distant metastasis remains the major cause of death from nasopharyngeal carcinoma (NPC). This study aimed to evaluate the clinical value of pretreatment serum lipid profiles in predicting clinical outcome of NPC.

Methodology I Principal Findings: A total of 1927 consecutive patients who had untreated NPC and completed radical IMRT between Jan. 2010 and Dec. 2011 were retrospectively reviewed. Pretreatment serum lipid indexes including total cholesterol, triglyceride, high-density lipoprotein cholesterol, low-density lipoprotein cholesterol, apolipoprotein A-I (apoAI) and apolipoprotein B were analyzed for their association with survivals, together with the clinical features (age, sex, pathological type, anemia, chemotherapy sequence and Epstein-Barr virus deoxyribonucleic acid). Hazard ratio (HR) and $95 \%$ confidence interval $(\mathrm{Cl})$ were calculated for each independent prognosticator. After univariate and multivariate survival analysis, low apoAI level $(<1.125 \mathrm{mmol} / \mathrm{L})$ appeared to predict poor 5 -year overall survival (OS), disease-free survival (DFS) and distant-metastasis-free survival (DMFS).The HRs were 1.549 (95\% Cl, 1.137-2.109), 1.293 (95\% Cl, 1.047-1.597) and 1.288 (95\% Cl, 1.022-1.623), respectively. Subgroup survival analysis showed that the apoAI maintained predicting independence for OS, DFS and DMFS in patients with locally advanced NPC, even in those treated with concurrent chemoradiotherapy.

Conclusions / Significance: NPC patient with low serum level of pretreatment apoAl might be at risk of distant metastasis. Treatment aiming to eradicate distant metastasis might improve survival of these patients.
\end{abstract}

Key words: nasopharyngeal carcinoma; apolipoprotein A-I; distant metastasis; survival; intensity-modulated radiotherapy

\section{Introduction}

The nasopharyngeal carcinoma (NPC) is a rare malignancy in the world, but it threats the health of many Chinese, especially in the southern provinces [1]. Multimodality treatments based on radiotherapy (RT) are now the standard management for NPC [2]. Owing to the popularization of the intensitymodulated radiotherapy (IMRT) technique, the overall survival (OS) of patients with NPC has now reached 85\% [3]. However, distant metastasis (DM) rather than local recurrence (LR) remains the major cause of death [2]. Even after intensified systemic chemotherapy, such as neoadjuvant chemotherapy (NACT) or adjuvant chemotherapy (ACT), nearly $15 \%$ of NPC patients still undergo DM after a 5-year follow-up $[4,5]$. In other words, there is a need to develop treatment modalities which could further 
reduce DM and improve survival in the era of IMRT.

Expression of a series of biomarkers, such as the epidermal growth factor receptor and the vascular endothelial growth factor, have been reported to be associated with progression of NPC and prognosis of the patients [6,7]. And antagonists and antibodies targeted those biomarkers have also been developed [8-10]. However, harsh detecting conditions of those markers and high cost of the targeted drugs limit their application. A marker which has simple testing methods and mature means of interference might be a target more practical for clinical treatment.

Serum lipid profiles are a group of practical indexes which can now be tested routinely and interfered easily in the daily clinical works [11,12]. Recent studies indicated level of serum lipids were predictors of oncogenesis, DM and long-term outcome of many malignant tumors, such as breast, lung, and hepatic cancers [13-15]. In patients with NPC, there are few studies focusing on the relationship between serum lipids and prognosis [16-19]. And in those studies, the RT technique was almost 2-dimensional radiotherapy (2DRT), or only one index of serum lipids was analyzed. Considering IMRT is now the main RT technique for NPC patients, this study aimed to evaluate the prognostic value of the serum lipid profiles in non-metastatic NPC patients who received IMRT.

\section{Materials and Methods}

\section{Ethical statement}

This study was approved by the Institutional Review Board of the Sun Yat-sen University Cancer Center. Written informed consents were obtained from all individual participants included in the study.

\section{Patient selection}

Patients with pathologically diagnosed and previously untreated NPC in our hospital from January 1st 2010 to December 31st 2011 were initially considered. The patients were enrolled into this study for the following reasons: (i) age $\geq 18$ and $<70$ years old; (ii) stage I-IVB disease at diagnosis; (iii) completed radical RT with IMRT technique. The exclusion criteria included: (i) DM before or during RT; (ii) Eastern Cooperative Oncology Group performance score $>2$; (iii) severe hematopoietic, heart, lung, liver or kidney dysfunctions unsuitable for RT; (iv) prior history of other malignancies; (v) lack of pretreatment serum lipid profiles, complete blood count or serum level of Epstein-Barr virus (EBV) deoxyribonucleic acid (DNA); (vi) regular intake of lipid-modulating drugs, such as niacins and statins, before treatment.

\section{Diagnosis and staging work-up}

Before treatment, all the patients underwent a biopsy under nasopharyngoscope to make the pathologic diagnosis. Then they received magnetic resonance imaging (MRI) of head and neck, thoraco-abdominal computed tomography (or chest radiograph plus abdominal ultrasonography), and whole-body bone scan (or positron emission tomography) to evaluate the stage. The stage of each patient was determined according to the 7th edition of TNM staging system of the Union for International Cancer Control / American Joint Cancer Committee [20].

\section{Serum lipid profiles}

Serum lipid profiles of each enrolled patient was tested a week before treatment started, including level of total cholesterol (TC), triglyceride (TG), high-density lipoprotein cholesterol (HDL-c), low-density lipoprotein cholesterol (LDL-c), apolipoprotein A-I (apoAI) and apolipoprotein B (apoB).

\section{Treatment}

Treatment strategy of all the patients was based on the guidelines of the National Comprehensive Cancer Network and our hospital. Stage T1-2N0M0 disease was treated with RT alone. Stage T2N1M0 and locally advanced-stage (stage III-IVB) diseases were treated with combination of RT and chemotherapy. The sequences of chemotherapy included concurrent chemotherapy (CCT) alone, NACT alone, NACT + $\mathrm{CCT}, \mathrm{CCT}+\mathrm{ACT}$, and NACT + CCT + ACT .

The irradiation technique of all the patients in this study was IMRT, whose target definition, delineation and dose prescription were made according to the International Commission on Radiation Units and Measurements Report 83 [21]. More details were in the supplementary materials.

The regimen of concurrent chemotherapy (CCT) was single-agent cisplatin $40 \mathrm{mg} / \mathrm{m}^{2}$ weekly or 80 $\mathrm{mg} / \mathrm{m}^{2}$ every 3 weeks throughout the whole procedure of RT. The NACT was administered every 3-4 weeks for 2-3 cycles, with the regimen comprised of docetaxel $75 \mathrm{mg} / \mathrm{m}^{2} \mathrm{~d} 1$ plus cisplatin $75 \mathrm{mg} / \mathrm{m}^{2} \mathrm{~d} 1$, or cisplatin $80 \mathrm{mg} / \mathrm{m}^{2}$ d1 plus 5-fluorouracil 1000 $\mathrm{mg} / \mathrm{m}^{2} \mathrm{~d} 1-4$. The ACT was performed every 3-4 weeks with the cisplatin plus 5-fluorouracil regimen, for 2-6 cycles.

\section{Follow-up}

The follow up after treatment was done by outpatient interview every 3-6 months for the first 3 years. During each interview, disease status and treatment toxicities were assessed through physical 
examination, head and neck MRI, chest radiograph, abdominal ultrasonography, hematology and biochemistry profiles, serum EBV DNA level. The whole-body bone scan was performed annually. In the 4 th and 5 th years, the patients were followed by outpatient interview, telephone and letters semi-annually. And the patients were followed annually thereafter, until death from NPC or December 31st 2016, whichever came first. The causes of deaths were confirmed by death certificates.

The primary endpoint of this study was OS. It referred to the percentage of the patients who were alive after a certain time period from pathological diagnosis. And secondary endpoints included disease-free survival (DFS), local-recurrence-free survival (LRFS) and distant-metastasis-free survival (DMFS). These 3 endpoints were defined as the percentage of patients who had no corresponding events after a certain time period from diagnosis. The events for DFS included death, LR and DM. And the events for LRFS and DMFS were LR and DM, respectively. The patient without death, LR or DM until December 31st 2016, and the patients lost to follow-up were regarded censored.

\section{Definitions of cutoff values}

The definition of anemia is based on the standard of the World Health Organization (WHO), in which anemia is defined as hemoglobin $\leq 130$ (male) or 120 (female) g/L [22]. The upper normal limit of pretreatment EBV DNA was 4000 copies/ml, which referred to the previous studies $[23,24]$. The cutoff value of the age was the median age of the patients involved. The cutoff values of the serum lipid profiles were determined by a receiver operating characteristic (ROC) analysis.

\section{Statistical analysis}

The survival analysis was performed to determine the prognostic factors. First, a univariate survival analysis based on Kaplan-Meier approach was used to screen the possible predictors from age, sex (female vs. male), T stage (T1-2 vs. T3-4), N stage (N0-1 vs. N2-3), pathologic type (WHO III vs. WHO I-II), CCT (yes vs. no), NACT/ACT (yes vs. no), anemia (no vs. yes), EBV DNA ( $\leq 4000$ vs. $>4000$ copies/ml), TC, TG, LDL-c, HDL-c, apoAI and apoB. Difference in survival of patients grouped by each factor above was assessed by a log-rank test.

The factors which showed statistical significance in univariate analysis then entered a multivariate analysis based on the Cox proportional hazards model, to determine their independence on prognosis prediction. The hazard ratio (HR) and 95\% confidence interval $(\mathrm{CI})$ of each independent risk factor were calculated.

Finally, the subgroup survival analyses of the serum lipid profiles which maintained as independent prognosticators were made in patients with early (stage I-II) and locally advanced (stage III-IVB) stages of disease, respectively. And subgroup analysis was also performed in those who had locally advanced NPC and received CCT.

The statistical analysis in this study was done through IBM SPSS Statistics 23.0 (IBM Co., Armonk, New York, US). A difference with a two-sided $P$ value of less than 0.05 was considered to be statistically significant. The whole procedure of the study was summarized as the Figure 1.

\section{Results}

\section{Patient profiles}

A total of 1927 patients were eligible for analysis, including 309 patients with early (stage I-II) diseases and 1618 patients with locally advanced (stage III-IVB) diseases. A total 121 patients were lost to follow-up (6.3\%). The median follow-up time was 66.2 (range, 5.97-89.7) months.

The baseline clinical characteristics of the patients were shown in Table 1 . The patients who received RT alone, $\mathrm{RT}+\mathrm{CCT}, \mathrm{NACT}+\mathrm{RT}, \mathrm{NACT}+$ $\mathrm{RT}+\mathrm{CCT}, \mathrm{RT}+\mathrm{CCT}+\mathrm{ACT}, \mathrm{NACT}+\mathrm{RT}+\mathrm{CCT}+$ ACT were 273, 676, 309, 633, 19 and 17, respectively.

\section{Cutoff values}

The median age of the patients was 45 (range, 18-70) years old. The cutoff values of TC, TG, HDL-c, LDL-c, apoAI and apoB for predicting OS were 4.845, $0.765,1.165,3.725,1.125$ and $0.995 \mathrm{mmol} / \mathrm{L}$, respectively. The ROC curves of the serum lipid indexes above were shown in Figure $S 1$ in the supplementary materials. Thus, the age ( $\leq 45$ vs. $>45$ years old $)$, TC $(\leq 4.845$ vs. $>4.845 \mathrm{mmol} / \mathrm{L}), \mathrm{TG}(\leq$ 0.765 vs. $>0.765 \mathrm{mmol} / \mathrm{L}), \mathrm{LDL}-\mathrm{c}(\leq 3.725$ vs. $>3.725$ $\mathrm{mmol} / \mathrm{L})$, HDL-c $(\geq 1.165$ vs. $<1.165 \mathrm{mmol} / \mathrm{L})$, apoAI $(\geq 1.125$ vs. $<1.125 \mathrm{mmol} / \mathrm{L})$ and apoB ( $\leq 0.995$ vs. $>$ $0.995 \mathrm{mmol} / \mathrm{L})$ were put into survival analysis, together with sex, $\mathrm{T}$ stage, $\mathrm{N}$ stage, pathologic type, CCT, NACT/ACT, anemia and EBV DNA.

\section{Survival analysis}

As shown in Table 1, the univariate analysis on the full group of patients showed that pathologic type, NACT/ACT and EBV DNA were possible predictors of the OS, DFS, LRFS and DMFS. Age, T stage, $\mathrm{N}$ stage, and apoAI were possible predictors of the OS, DFS and DMFS. And sex was a possible predictor of the OS and DMFS. The possible predictors of OS also included HDL-c, LDL-c and apoB. 


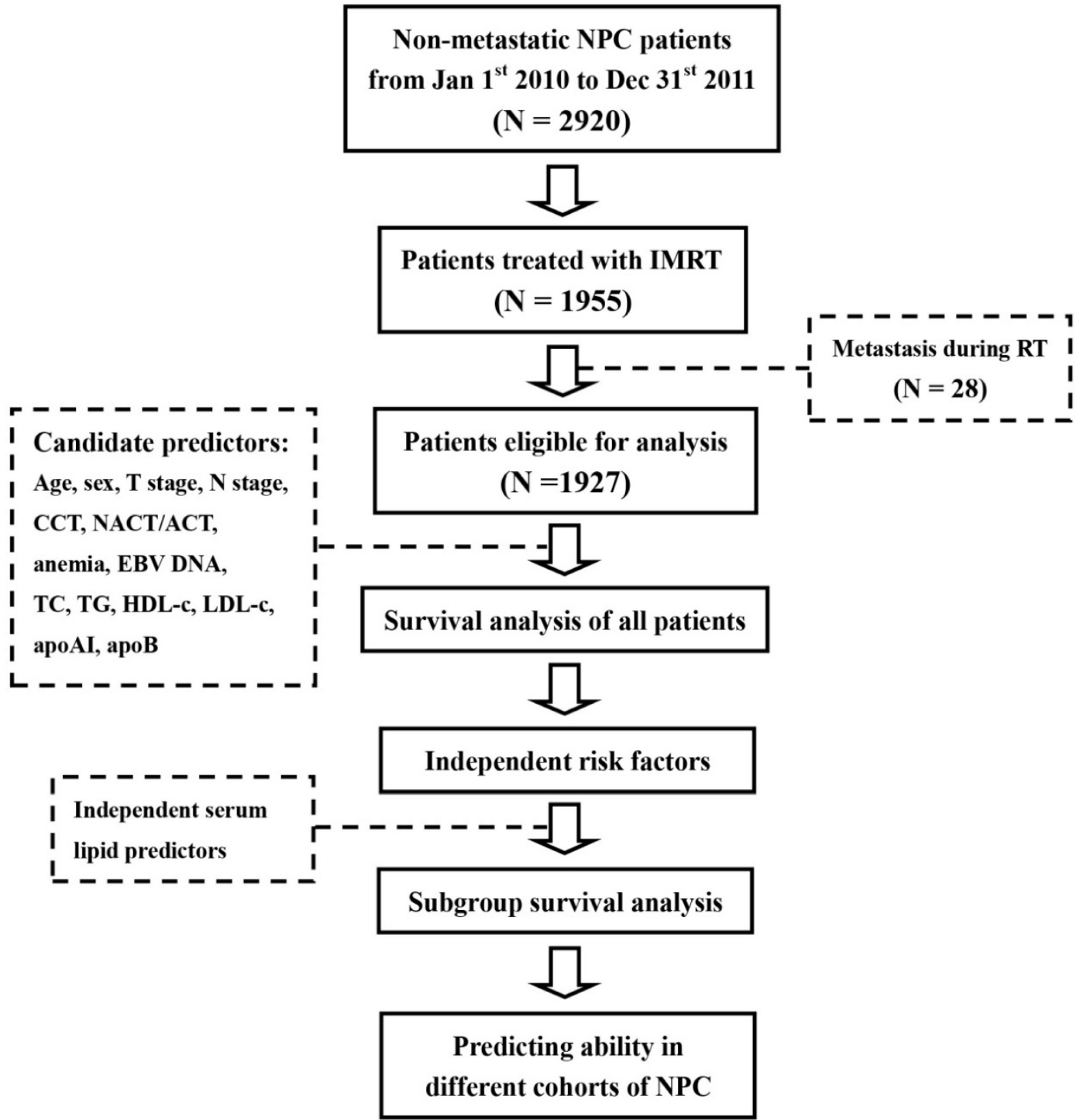

Figure 1. Procedure of this study. Abbreviations: NPC, nasopharyngeal carcinoma; RT, radiation therapy; IMRT, intensity-modulated radiotherapy; CCT, concurrent chemotherapy; NACT, neoadjuvant chemotherapy; ACT, adjuvant chemotherapy; TC, total cholesterol, TG, triglyceride; HDL-c, high-density lipoprotein cholesterol; LDL-c, low-density lipoprotein cholesterol; apoAl, apolipoprotein A-l; apoB, apolipoprotein B; EBV DNA, Epstein-Barr virus deoxyribonucleic acid.

Table 1. Univariate survival analysis in the full group of patients.

\begin{tabular}{|c|c|c|c|c|c|c|c|c|}
\hline & OS & $P$ Value & DFS & $P$ Value & LRFS & $P$ Value & DMFS & $P$ Value \\
\hline All Patients & $\begin{array}{l}1684 / 1927 \\
(87.4 \%)\end{array}$ & & $\begin{array}{l}1566 / 1927 \\
(81.3 \%)\end{array}$ & & $\begin{array}{l}1808 / 1927 \\
(93.8 \%)\end{array}$ & & $1628 / 1927(84.5 \%)$ & \\
\hline \multicolumn{9}{|c|}{ Age (years old) } \\
\hline$\leq 45$ & $826 / 917(90.1 \%)$ & $0.001^{* *}$ & $\begin{array}{l}768 / 917 \\
(83.8 \%)\end{array}$ & $0.009^{* *}$ & $865 / 917(94.3 \%)$ & 0.364 & 799/917 (87.1\%) & $0.002^{* *}$ \\
\hline$>45$ & $858 / 1010(85.0 \%)$ & & $\begin{array}{l}798 / 1010 \\
(79.0 \%)\end{array}$ & & $943 / 1010(93.4 \%)$ & & $829 / 1010(82.1 \%)$ & \\
\hline \multicolumn{9}{|l|}{ Sex } \\
\hline Female & $450 / 501(89.8 \%)$ & $0.049^{*}$ & $\begin{array}{l}414 / 501 \\
(82.6 \%)\end{array}$ & 0.347 & $465 / 501(92.8 \%)$ & 0.311 & $440 / 501(87.8 \%)$ & $0.016^{*}$ \\
\hline Male & $\begin{array}{l}1234 / 1426 \\
(86.5 \%)\end{array}$ & & $\begin{array}{l}1152 / 1426 \\
(80.8 \%)\end{array}$ & & $\begin{array}{l}1343 / 1426 \\
(94.2 \%)\end{array}$ & & $1188 / 1426(83.3 \%)$ & \\
\hline \multicolumn{9}{|l|}{ T Stage } \\
\hline T1-2 & $509 / 550(92.5 \%)$ & $<0.001^{* *}$ & $\begin{array}{l}479 / 550 \\
(87.1 \%)\end{array}$ & $<0.001^{* *}$ & $516 / 550(93.8 \%)$ & 0.884 & $498 / 550(90.5 \%)$ & $<0.001^{* *}$ \\
\hline T3-4 & $\begin{array}{l}1175 / 1377 \\
(85.3 \%)\end{array}$ & & $\begin{array}{l}1087 / 1377 \\
(78.9 \%)\end{array}$ & & $\begin{array}{l}1292 / 1377 \\
(93.8 \%)\end{array}$ & & $1130 / 1377(82.1 \%)$ & \\
\hline \multicolumn{9}{|l|}{ N Stage } \\
\hline N0-1 & $966 / 1048(92.2 \%)$ & $<0.001^{* *}$ & $902 / 1048$ & $<0.001^{* *}$ & 988/1048 (94.3\%) & 0.219 & 934/1048 (89.1\%) & $<0.001^{* *}$ \\
\hline
\end{tabular}




\begin{tabular}{|c|c|c|c|c|c|c|c|c|}
\hline & OS & $P$ Value & DFS & $P$ Value & LRFS & $P$ Value & DMFS & $P$ Value \\
\hline & & & $(86.1 \%)$ & & & & & \\
\hline N2-3 & $718 / 879(81.7 \%)$ & & $\begin{array}{l}644 / 879 \\
(75.5 \%)\end{array}$ & & $820 / 879(93.3 \%)$ & & $694 / 879(79.0 \%)$ & \\
\hline \multicolumn{9}{|c|}{ Pathologic type } \\
\hline WHO III & $\begin{array}{l}1625 / 1849 \\
(87.9 \%)\end{array}$ & $0.002^{* *}$ & $\begin{array}{l}1512 / 1849 \\
(81.8 \%)\end{array}$ & $0.007^{* *}$ & $\begin{array}{l}1741 / 1849 \\
(94.2 \%)\end{array}$ & $0.002^{* *}$ & $1571 / 1849(85.0 \%)$ & $0.007^{* *}$ \\
\hline WHO I-II & $59 / 78(75.6 \%)$ & & $54 / 78(69.2 \%)$ & & $67 / 78(85.9 \%)$ & & $57 / 78(73.1 \%)$ & \\
\hline \multicolumn{9}{|l|}{ CCT } \\
\hline Yes & $508 / 581(87.4 \%)$ & 0.951 & $\begin{array}{l}479 / 581 \\
(80.8 \%)\end{array}$ & 0.446 & $547 / 581(93.7 \%)$ & 0.714 & $496 / 581(84.1 \%)$ & 0.532 \\
\hline No & $\begin{array}{l}1176 / 1346 \\
(87.4 \%)\end{array}$ & & $\begin{array}{l}1087 / 1346 \\
(82.4 \%)\end{array}$ & & $\begin{array}{l}1261 / 1346 \\
(94.1 \%)\end{array}$ & & $1132 / 1346(85.4 \%)$ & \\
\hline \multicolumn{9}{|c|}{ NACT/ACT } \\
\hline Yes & $865 / 949(83.7 \%)$ & $<0.001^{* *}$ & $\begin{array}{l}812 / 949 \\
(77.1 \%)\end{array}$ & $<0.001^{* *}$ & $906 / 949(95.5 \%)$ & $0.002^{* *}$ & $837 / 949(80.9 \%)$ & $<0.001^{\star *}$ \\
\hline No & $819 / 978(91.1 \%)$ & & $\begin{array}{l}754 / 978 \\
(85.6 \%)\end{array}$ & & $902 / 978(92.2 \%)$ & & $791 / 978(88.2 \%)$ & \\
\hline \multicolumn{9}{|l|}{ Anemia } \\
\hline Yes & $\begin{array}{l}1531 / 1745 \\
(87.7 \%)\end{array}$ & 0.172 & $\begin{array}{l}1424 / 1745 \\
(81.6 \%)\end{array}$ & 0.269 & $\begin{array}{l}1635 / 1745 \\
(93.7 \%)\end{array}$ & 0.481 & $1480 / 1745(84.8 \%)$ & 0.226 \\
\hline No & $153 / 182(84.1 \%)$ & & $\begin{array}{l}142 / 182 \\
(78.0 \%)\end{array}$ & & $173 / 182(95.1 \%)$ & & $148 / 182(81.8 \%)$ & \\
\hline \multicolumn{9}{|c|}{ EBV DNA (copies/ml) } \\
\hline$\leq 4000$ & $\begin{array}{l}1061 / 1151 \\
(92.2 \%)\end{array}$ & $<0.001^{* *}$ & $\begin{array}{l}1015 / 1151 \\
(88.2 \%)\end{array}$ & $<0.001^{* *}$ & $\begin{array}{l}1102 / 1151 \\
(95.7 \%)\end{array}$ & $<0.001^{* *}$ & $1044 / 1151(90.7 \%)$ & $<0.001^{* *}$ \\
\hline$>4000$ & $623 / 776(80.3 \%)$ & & $\begin{array}{l}551 / 776 \\
(71.0 \%)\end{array}$ & & $706 / 776(91.0 \%)$ & & $584 / 776(75.3 \%)$ & \\
\hline \multicolumn{9}{|c|}{ TC (mmol/L) } \\
\hline$\leq 4.845$ & $644 / 722(89.2 \%)$ & 0.054 & $\begin{array}{l}594 / 722 \\
(82.3 \%)\end{array}$ & 0.316 & $674 / 722(93.4 \%)$ & 0.618 & $619 / 722(85.7 \%)$ & 0.197 \\
\hline$>4.845$ & $\begin{array}{l}1040 / 1205 \\
(86.3 \%)\end{array}$ & & $\begin{array}{l}972 / 1205 \\
(80.7 \%)\end{array}$ & & $\begin{array}{l}1134 / 1205 \\
(94.1 \%)\end{array}$ & & $1009 / 1205(83.7 \%)$ & \\
\hline \multicolumn{9}{|c|}{ TG (mmol/L) } \\
\hline$\leq 0.765$ & $235 / 259(90.7 \%)$ & 0.091 & $\begin{array}{l}217 / 259 \\
(83.8 \%)\end{array}$ & 0.260 & $244 / 259(94.2 \%)$ & 0.740 & $227 / 259(87.6 \%)$ & 0.136 \\
\hline$>0.765$ & $1449 / 1668(86.9 \%)$ & & $\begin{array}{l}1349 / 1668 \\
(80.9 \%)\end{array}$ & & $\begin{array}{l}1564 / 1668 \\
(93.8 \%)\end{array}$ & & $1401 / 1668(84.0 \%)$ & \\
\hline \multicolumn{9}{|c|}{ HDL-c (mmol/L) } \\
\hline$\geq 1.165$ & $\begin{array}{l}1034 / 1166 \\
(88.7 \%)\end{array}$ & $0.025^{*}$ & $\begin{array}{l}957 / 1166 \\
(82.1 \%)\end{array}$ & 0.243 & $\begin{array}{l}1098 / 1166 \\
(94.2 \%)\end{array}$ & 0.422 & $996 / 1166(85.4 \%)$ & 0.138 \\
\hline$<1.165$ & $650 / 761(85.4 \%)$ & & $\begin{array}{l}609 / 761 \\
(80.0 \%)\end{array}$ & & $710 / 761(93.3 \%)$ & & $632 / 761(83.0 \%)$ & \\
\hline \multicolumn{9}{|c|}{ LDL-c (mmol/L) } \\
\hline$\leq 3.725$ & $\begin{array}{l}1234 / 1399 \\
(88.2 \%)\end{array}$ & $0.045^{*}$ & $\begin{array}{l}1148 / 1399 \\
(82.1 \%)\end{array}$ & 0.100 & $\begin{array}{l}1310 / 1399 \\
(93.6 \%)\end{array}$ & 0.702 & $1191 / 1399(85.1 \%)$ & 0.136 \\
\hline$>3.725$ & $450 / 528(85.2 \%)$ & & $\begin{array}{l}418 / 528 \\
(79.2 \%)\end{array}$ & & $498 / 528(94.3 \%)$ & & $437 / 528(82.8 \%)$ & \\
\hline \multicolumn{9}{|c|}{ ApoAI (mmol/L) } \\
\hline$\geq 1.125$ & $\begin{array}{l}1042 / 1160 \\
(89.8 \%)\end{array}$ & $<0.001^{* *}$ & $\begin{array}{l}972 / 1160 \\
(83.8 \%)\end{array}$ & $0.001^{* *}$ & $\begin{array}{l}1093 / 1160 \\
(94.2 \%)\end{array}$ & 0.369 & $1007 / 1160(86.8 \%)$ & $0.001^{*}$ \\
\hline$<1.125$ & $642 / 767(83.7 \%)$ & & $\begin{array}{l}594 / 767 \\
(77.4 \%)\end{array}$ & & $715 / 767(93.2 \%)$ & & $621 / 767(81.0 \%)$ & \\
\hline \multicolumn{9}{|c|}{ ApoB (mmol/L) } \\
\hline$\leq 0.995$ & $\begin{array}{l}1024 / 1149 \\
(89.1 \%)\end{array}$ & $0.005^{* *}$ & $\begin{array}{l}948 / 1149 \\
(82.5 \%)\end{array}$ & 0.079 & $\begin{array}{l}1082 / 1149 \\
(94.2 \%)\end{array}$ & 0.372 & $984 / 1149(85.6 \%)$ & 0.078 \\
\hline$>0.995$ & $660 / 778(84.8 \%)$ & & $\begin{array}{l}618 / 778 \\
(79.4 \%)\end{array}$ & & $726 / 778(93.3 \%)$ & & $644 / 778(82.8 \%)$ & \\
\hline
\end{tabular}

${ }^{*} P<0.05,{ }^{* *} P<0.01$. Abbreviations: OS, overall survival; DFS, disease-free survival; LRFS, local-recurrence-free survival; DMFS, distant-metastasis-free survival; WHO, World Health Organization; CCT, concurrent chemotherapy; NACT, neoadjuvant chemotherapy; ACT, adjuvant chemotherapy; EBV DNA, Epstein-Barr virus deoxyribonucleic acid; TC, total cholesterol, TG, triglyceride; HDL-c, high-density lipoprotein cholesterol; LDL-c, low-density lipoprotein cholesterol; apoAI, apolipoprotein A-I; apoB, apolipoprotein B.

The results of multivariate analysis were shown in Table 2. WHO pathologic type I-II and EBV DNA > 4000 copies/ $\mathrm{ml}$ were independent risk factors of the OS, DFS, LRFS and DMFS. And age $>45$ years old, T3-4 disease and N2-3 disease were independent risk factors of the OS, DFS and DMFS. Among the serum lipids, apoAI $<1.125 \mathrm{mmol} / \mathrm{L}$ appeared to predict poor OS $(\mathrm{HR}=1.594,95 \% \mathrm{CI}=1.137-2.109)$, DFS $(\mathrm{HR}$ $=1.293,95 \% \mathrm{CI}=1.047-1.597)$ and DMFS $(\mathrm{HR}=1.288$, $95 \% \mathrm{CI}=1.022-1.623$ ) independently.

To explore the prognostic impact of apoAI on different stages of NPC, the subgroup survival analyses were performed in cases with early and locally advanced diseases, respectively. The results 
were shown as Table S1-S3 (refer to the supplementary materials). Although apoAI was not a statistically significant predictor of patients with stage I-II NPC, the subgroup analysis in patients with stage III-IV NPC revealed that apoAI $<1.125 \mathrm{mmol} / \mathrm{L}$ still predicted poorer OS, DFS and DMFS independently (HRs were 1.511, 1.321 and 1.401, respectively). Considering IMRT plus CCT was the standard treating mode for stage III-IVB NPC, we also performed subgroup analysis in stage III-IV NPC patients treated with CCT. ApoAI $<1.125 \mathrm{mmol} / \mathrm{L}$ maintained to predict poorer OS, DFS and DMFS independently (HRs were 1.548, 1.277 and 1.383, respectively). The OS curves of patients divided by the apoAI in full group and subgroup analyses were shown in Figure 2. The DFS and DMFS curves were shown as Figure S2 and S3, respectively.

\section{Discussion}

Because of improved treatment effects and decreased adverse events, IMRT has replaced the 2DRT and 3-dimensional RT as the standard RT technique for NPC [25]. The advent of CCT further reduces the LR and ameliorates the OS of locally advanced NPC [26]. But until now, the DM still threats the survival of NPC patients, especially those with locally advanced diseases. In this study, the 5-year DM rate of 1927 patients treated with IMRT appeared to be $15.5 \%$, which was similar to the data reported by the previous studies [4,5]. In 1618 patients with stage III-IVB diseases, the DM rate was as high as $17.4 \%(16.8 \%$ in cases treated with standard IMRT plus CCT). To reduce DM and achieve final success of treatment, approaches were made to change the chemotherapy sequence into NACT plus CCT, or CCT plus ACT. Nevertheless, there was no confirmed evidence supporting that addition of NACT or ACT brought survival benefit $[27,28]$. Considering increased incidence of severe toxicities [29], the clinical application of NACT and ACT are now limited. Some oncologists tried to explore indexes indicating patients who might be at high risk of DM and in a real need of such intensified modes of chemotherapy. For example, stage N2-3 diseases and high serum level of pretreatment EBV DNA are acknowledged risk factors predicting DM $[23,24]$.

Table 2. Multivariate survival analysis in the full group of patients.

\begin{tabular}{|c|c|c|c|c|c|}
\hline Survival & Factors & Variables & $P$ Value & HR & $95 \% \mathrm{CI}$ for $\mathrm{HR}$ \\
\hline \multirow[t]{11}{*}{ OS } & Age & $>45$ vs. $\leq 45$ years old & $0.001^{* *}$ & 1.540 & $1.181-2.008$ \\
\hline & Sex & Male vs. Female & 0.572 & 1.097 & $0.796-1.513$ \\
\hline & T stage & T3-4 vs. T1-2 & $0.001^{* *}$ & 1.763 & $1.248-2.491$ \\
\hline & N stage & N2-3 vs. N0-1 & $<0.001^{* *}$ & 1.943 & $1.453-2.600$ \\
\hline & Pathologic type & WHO I-II vs. III & $0.003^{* *}$ & 2.062 & $1.284-3.313$ \\
\hline & NACT / ACT & Yes vs. No & 0.384 & 1.135 & $0.853-1.510$ \\
\hline & EBV DNA & $>4000$ vs. $\leq 4000$ copies $/ \mathrm{ml}$ & $<0.001^{* *}$ & 2.023 & $1.527-2.679$ \\
\hline & HDL-c & $<1.165$ vs. $\geq 1.165 \mathrm{mmol} / \mathrm{L}$ & 0.621 & 0.925 & $0.678-1.262$ \\
\hline & LDL-c & $>3.725$ vs. $\leq 3.725 \mathrm{mmol} / \mathrm{L}$ & 0.594 & 1.101 & $0.773-1.567$ \\
\hline & apoAI & $<1.125$ vs. $\geq 1.125 \mathrm{mmol} / \mathrm{L}$ & $0.005^{* *}$ & 1.549 & $1.137-2.109$ \\
\hline & apoB & $>0.995$ vs. $\leq 0.995 \mathrm{mmol} / \mathrm{L}$ & 0.134 & 1.287 & $0.925-1.792$ \\
\hline \multirow[t]{8}{*}{ DFS } & Age & $>45$ vs. $\leq 45$ years old & $0.013^{*}$ & 1.308 & $1.059-1.615$ \\
\hline & Sex & Male vs. Female & 0.796 & 0.968 & $0.757-1.238$ \\
\hline & T stage & T3-4 vs. T1-2 & $0.006^{* *}$ & 1.458 & $1.114-1.909$ \\
\hline & N stage & N2-3 vs. N0-1 & $0.005^{* *}$ & 1.391 & $1.103-1.755$ \\
\hline & Pathologic type & WHO I-II vs. III & $0.004^{* *}$ & 1.843 & $1.215-2.795$ \\
\hline & NACT/ACT & Yes vs. No & 0.508 & 1.082 & $0.857-1.365$ \\
\hline & EBV DNA & $>4000$ vs. $\leq 4000$ copies $/ \mathrm{ml}$ & $<0.001^{* *}$ & 2.356 & $1.870-2.968$ \\
\hline & apoAI & $<1.125$ vs. $\geq 1.125 \mathrm{mmol} / \mathrm{L}$ & $0.017^{*}$ & 1.293 & $1.047-1.597$ \\
\hline \multirow[t]{3}{*}{ LRFS } & Pathologic type & WHO I-II vs. III & $0.002^{* *}$ & 2.664 & $1.431-4.961$ \\
\hline & NACT/ACT & Yes vs. No & 0.056 & 1.458 & $0.990-2.148$ \\
\hline & EBV DNA & $>4000$ vs. $\leq 4000$ copies $/ \mathrm{ml}$ & $<0.001^{* *}$ & 2.140 & $1.464-3.130$ \\
\hline \multirow[t]{8}{*}{ DMFS } & Age & $>45$ vs. $\leq 45$ years old & $0.003^{* *}$ & 1.427 & $1.130-1.803$ \\
\hline & Sex & Male vs. Female & 0.220 & 1.197 & $0.898-1.594$ \\
\hline & T stage & T3-4 vs. T1-2 & $0.001^{* *}$ & 1.718 & $1.262-2.339$ \\
\hline & N stage & N2-3 vs. N0-1 & $0.001^{* *}$ & 1.553 & $1.202-2.006$ \\
\hline & Pathologic type & WHO I-II vs. III & $0.007^{* *}$ & 1.854 & $1.186-2.897$ \\
\hline & NACT/ACT & Yes vs. No & 0.871 & 1.021 & $0.793-1.315$ \\
\hline & EBV DNA & $>4000$ vs. $\leq 4000$ copies $/ \mathrm{ml}$ & $<0.001^{* *}$ & 2.432 & $1.883-3.141$ \\
\hline & apoAI & $<1.125$ vs. $\geq 1.125 \mathrm{mmol} / \mathrm{L}$ & $0.032^{*}$ & 1.288 & $1.022-1.623$ \\
\hline
\end{tabular}

${ }^{*} P<0.05,{ }^{* *} P<0.01$. Abbreviations: HR, hazard ratio; CI, confidence interval; OS, overall survival; DFS, disease-free survival; LRFS, local-recurrence-free survival; DMFS, distant-metastasis-free survival; WHO, World Health Organization; CCT, concurrent chemotherapy; NACT, neoadjuvant chemotherapy; ACT, adjuvant chemotherapy; EBV DNA, Epstein-Barr virus deoxyribonucleic acid; TC, total cholesterol, TG, triglyceride; HDL-c, high-density lipoprotein cholesterol; LDL-c, low-density lipoprotein cholesterol; apoAI, apolipoprotein A-I; apoB, apolipoprotein B. 


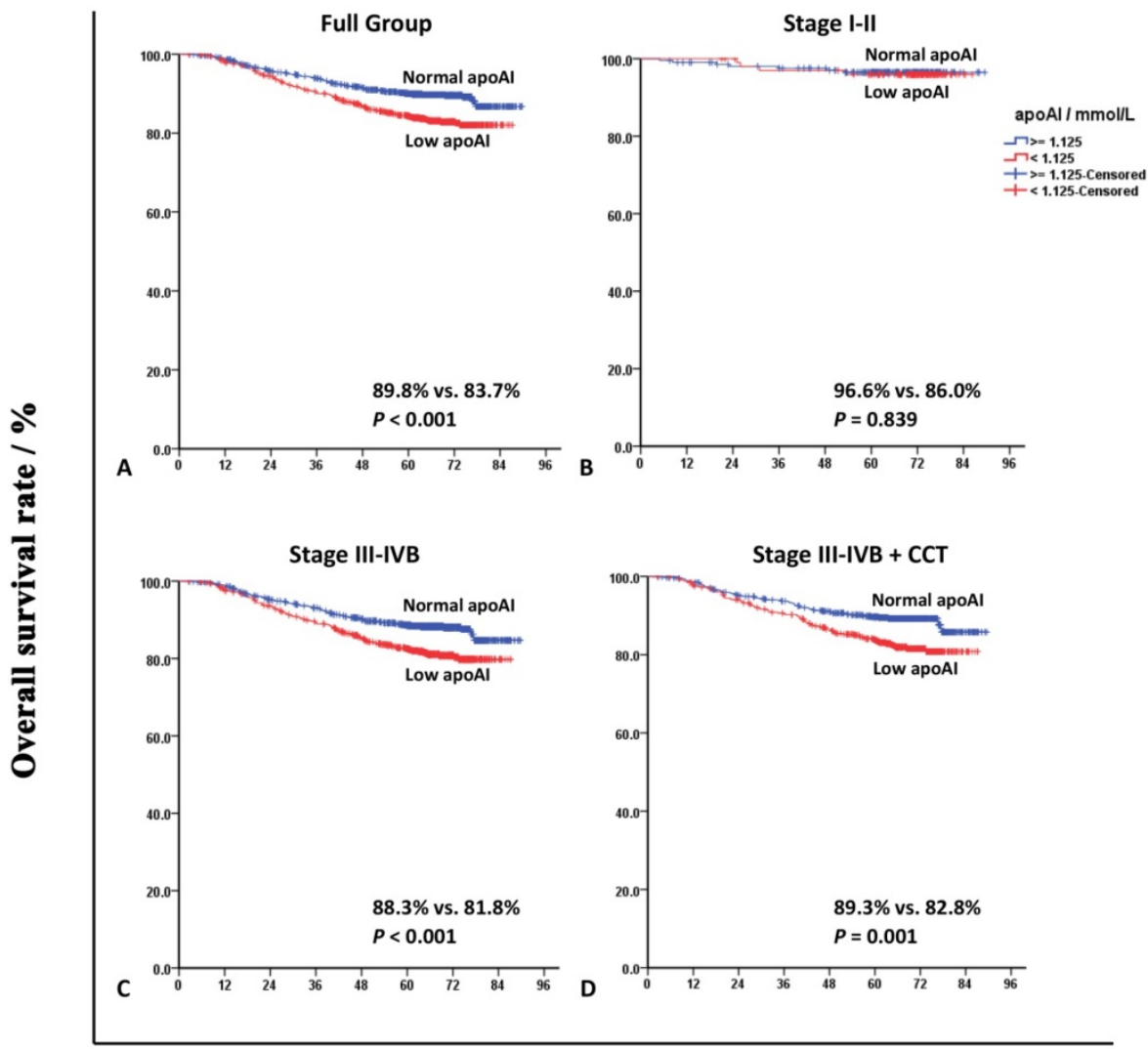

Time after diagnosis / Month

Figure 2. Overall survival curves of patients grouped by the apolipoprotein A-I (apoAl) in full group and subgroup analyses. Panel A: Full group of patients. Panel B: Patients with stage I-II nasopharyngeal carcinoma (NPC). Panel C: Patients with stage III-IVB NPC. Panel D: Patients with stage III-IVB NPC and concurrent chemotherapy (CCT) during radiotherapy.

The role of lipid metabolism in cancer development and treatment is one of the research hotspots nowadays. The prognostic value of the serum lipids, especially the apoAI, was shown in many kinds of cancers [15,29,30]. Recent studies of Jiang et al and Luo et al reported apoAI as a prognostic factor of NPC in both non-metastatic and metastatic patients who received 2DRT, respectively $[16,17]$. For validating the results in the era of IMRT, we performed an analysis on correlation between all commonly used serum lipid indexes and prognosis of NPC, in a large scale of cases treated with IMRT. This was the strength of our study. In this study, the cutoff values of pretreatment serum TC, TG, HDL-c, LDL-c, apoAI and apoB were calculated as $4.845,0.765,1.165$, $3.725,1.125$ and $0.995 \mathrm{mmol} / \mathrm{L}$, respectively. But through univariate and multivariate survival analyses, only low apoAI $(<1.125 \mathrm{mmol} / \mathrm{L})$ emerged as a factor predicting the poor DMFS and OS (HRs were 1.288 and 1.594 , respectively). Its predicting abilities were independent of the well-known prognosticators, such as the $\mathrm{N}$ stage and EBV DNA. Subgroup analyses revealed that the prognostic impact of apoAI mainly existed in stage III-IVB NPC patients but not the ones with stage I-II diseases. In cases treated with CCT, low apoAI maintained its inverse association with DMFS and OS. Based on these results, it could be easily inferred that low level of serum apoAI before treatment might be an indicator of intensified chemotherapy for patients with locally advanced NPC, even treated with IMRT and CCT. Although HDL-c finally failed to be an independent predictor, low level of HDL-c was associated with decreased $O S$ in the univariate analysis. The explanation might be that the concentration of HDL-c was influenced by apoAI, which was the core component of HDL-c [31]. However, Liu et al gained an opposite result that high HDL-c level predicted poor clinical outcome [18]. And additionally, the study of Tang et al showed a negative correlation between serum LDL-c level and survival of NPC patients [19].

ApoAI might not predict the DM only through reflecting the tumor burden, as N stage and EBV DNA did. Its predicting abilities might also come from its anti-tumor activities. It have been widely demonstrated in animal models that apoAI could inhibit tumor progression. The studies of 
Zamanian-Daryoush et al and Su et al demonstrated that apoAI gene null mice had obviously larger size of the xenograft tumor and poorer survival. Supplement of exogenous apoAI resulted in not only the retardation of tumor growth but also the tumor shrinkage [32,33]. Until now, the exact molecular mechanisms through which apoAI plays its anti-tumor role remain unclear. Yet apoAI and its mimetic peptides was proved to alter the microenvironment to a state against growth of the tumor cells, including decreasing levels of the anti-apoptotic protein survivin, reducing recruitment of myeloid-derived suppressor cells, and increasing accumulation of tumor-associated macrophages with an M1-like anti-tumor phenotype and CD8+ T cells [32-34]. ApoA1 could also inversely influence the migration of the tumor cells, through decreasing concentration and activity of the migration-related enzyme matrix metalloproteinase-9, and reducing the tumor angiogenesis [32,35]. And it could also inhibit inflammation which was thought to take an important part in tumor metastasis [36]. For NPC, a recent study of Zheng et al showed that apoAI biomimetic peptides could inhibited the growth of 5-8F cells both in vitro and in vivo [37]. In a word, apoAI might be a potential treatment target of malignant tumors, including NPC. Actually, the drugs used to increase the serum apoAI concentration, such as the niacin and statin [38,39], were also tried to prevent or treat malignant tumors. Park et al demonstrated in 114116 cases of healthy individuals that niacin intake could reduce the risk of skin squamous cell carcinoma [40]. And several studies proved that statin intake could improve survivals of colon and overian cancers [41,42]. These lipid-modulating agents might be another choice which could help to further eradicate the DM of the locally advanced NPC patients, when combined with chemoradiotherapy.

\section{Conclusion}

In conclusion, this study proved pretreatment serum apoAI level $<1.125 \mathrm{mmol} / \mathrm{L}$ as an independent risk factor to predict DM and death of NPC patients, particular those with stage III-IVB diseases. This finding may be informative for clinicians to conduct clinical trials on new therapeutic modalities, and direct individualized treatment strategies. Indeed, the retrospective nature was the main disadvantage of this study. Therefore, we informed that further verification might be needed before the results of this study could be popularized.

\section{Abbreviations}

NPC: nasopharyngeal carcinoma; RT: radiotherapy; IMRT: intensity-modulated radiother- apy; OS: overall survival; DM: distant metastasis; LR: local recurrence; NACT: neoadjuvant chemotherapy; ACT: adjuvant chemotherapy; 2DRT: 2-dimensional radiotherapy; EBV: Epstein-Barr virus; DNA: deoxyribonucleic acid; MRI: magnetic resonance imaging; TC: total cholesterol; TG: triglyceride; HDL-c: high-density lipoprotein cholesterol; LDL-c: low-density lipoprotein cholesterol; apoAI: apolipoprotein A-I; apoB: apolipoprotein B; CCT: concurrent chemotherapy; DFS: disease-free survival; LRFS: local-recurrence-free survival; DMFS: distantmetastasis-free survival; WHO: World Health Organization; ROC: receiver operating characteristic; HR: hazard ratio; CI: confidence interval.

\section{Supplementary Material}

Supplementary figures and tables. http://www.jcancer.org/v09p0702s1.pdf

\section{Competing Interests}

The authors have declared that no competing interest exists.

\section{References}

1. Chen W, Zheng R, Baade PD, et al. Cancer statistics in China, 2015. CA Cancer J Clin. 2016; 66(2): 115-132.

2. Chua ML, Wee JT, Hui EP, Chan AT. Nasopharyngeal carcinoma. Lancet. 2016; 387(10022): 1012-1024

3. Lee AW, Ng WT, Chan LL, et al. Evolution of treatment for nasopharyngeal cancer--success and setback in the intensity-modulated radiotherapy era. Radiother Oncol. 2014; 110(3): 377-384.

4. Li AC, Xiao WW, Shen GZ, et al. Distant metastasis risk and patterns of nasopharyngeal carcinoma in the era of IMRT: long-term results and benefits of chemotherapy. Oncotarget. 2015; 6(27): 24511-24521.

5. Kang M, Zhou P, Wei T, et al. A new $\mathrm{T}$ staging system for nasopharyngeal carcinoma based on intensity-modulated radiation therapy: results from a prospective multicentric clinical study. Am J Cancer Res. 2017; 7(2): 346-356.

6. Zheng LS, Yang JP, Cao Y, et al. SPINK6 Promotes Metastasis of Nasopharyngeal Carcinoma via Binding and Activation of Epithelial Growth Factor Receptor. Cancer Res. 2017; 77(2): 579-589.

7. Law EW, Cheung AK, Kashuba VI, et al. Anti-angiogenic and tumor-suppressive roles of candidate tumor-suppressor gene, Fibulin-2, in nasopharyngeal carcinoma. Oncogene. 2012; 31(6): 728-738.

8. You R, Hua YJ, Liu YP, et al. Concurrent Chemoradiotherapy with or without Anti-EGFR-Targeted Treatment for Stage II-IVb Nasopharyngeal Carcinoma: Retrospective Analysis with a Large Cohort and Long Follow-up. Theranostics. 2017; 7(8): 2314-2324.

9. Xu T, Liu Y, Dou S, et al. Weekly cetuximab concurrent with IMRT aggravated radiation-induced oral mucositis in locally advanced nasopharyngeal carcinoma: Results of a randomized phase II study. Oral Oncol. 2015; 51(9): 875-879.

10. Lee NY, Zhang Q, Pfister DG, et al. Addition of bevacizumab to standard chemoradiation for locoregionally advanced nasopharyngeal carcinoma (RTOG 0615): a phase 2 multi-institutional trial. Lancet Oncol. 2012; 13(2):172-180

11. Kingwell BA, Chapman MJ, Kontush A, Miller NE. HDL-targeted therapies: progress, failures and future. Nat Rev Drug Discov. 2014; 13(6): 445-464.

12. Silverman MG, Ference B, Im K, et al. Association Between Lowering LDL-C and Cardiovascular Risk Reduction Among Different Therapeutic Interventions: A Systematic Review and Meta-analysis. JAMA. 2016; 316(12): 1289-1297.

13. Berrino F, Villarini A, Traina A, et al. Metabolic syndrome and breast cancer prognosis. Breast Cancer Res Treat. 2014; 147(1): 159-165.

14. Zhou T, Zhan J, Fang W, et al. Serum low-density lipoprotein and low-density lipoprotein expression level at diagnosis are favorable prognostic factors in patients with small-cell lung cancer (SCLC). BMC Cancer. 2017; 17(1): 269.

15. Ma XL, Gao XH, Gong ZJ, et al. Apolipoprotein A1: a novel serum biomarker for predicting the prognosis of hepatocellular carcinoma after curative resection. Oncotarget. 2016; 7(43): 70654-70668.

16. Jiang R, Yang $\mathrm{ZH}$, Luo DH, et al. Elevated apolipoprotein A-I levels are associated with favorable prognosis in metastatic nasopharyngeal carcinoma. Med Oncol. 2014; 31(8): 80. 
17. Luo XL, Zhong GZ, Hu LY, et al. Serum apolipoprotein A-I is a novel prognostic indicator for non-metastatic nasopharyngeal carcinoma. Oncotarget. 2015; 6(41): 44037-44048.

18. Liu YY, Lin SJ, Chen YY, et al. High-density lipoprotein cholesterol as a predictor of poor survival in patients with nasopharyngeal carcinoma. Oncotarget. 2016; 7(28): 42978-42987.

19. Tang Q, Hu QY, Piao YF, Hua YH. Correlation between pretreatment serum LDL-cholesterol levels and prognosis in nasopharyngeal carcinoma patients. Onoco Targets Ther. 2016; 9: 2585-2591.

20. OuYang PY, Su Z, Ma XH, et al. Comparison of TNM staging systems for nasopharyngeal carcinoma, and proposal of a new staging system. Br J Cancer. 2013; 109(12): 2987-2997.

21. ICRU Report. Vol. 83: Prescribing, Recording, and Reporting Photon-Beam Intensity-Modulated Radiation Therapy (IMRT). Maryland: International Commission on Radiation Units and Measurements; 2010.

22. The Global Prevalence Of Anaemia In 2011. Geneva Switzerland WHO. 2011; 126(11): 5409-5418.

23. Tang LQ, Chen QY, Fan W, et al. Prospective study of tailoring whole-body dual-modality [18F] fluorodeoxyglucose positron emission tomography/computed tomography with plasma Epstein-Barr virus DNA for detecting distant metastasis in endemic nasopharyngeal carcinoma at initial staging. J Clin Oncol. 2013; 31(23): 2861-2869.

24. Guo SS, Tang LQ, Zhang L, et al. The impact of the cumulative dose of cisplatin during concurrent chemoradiotherapy on the clinical outcomes of patients with advanced-stage nasopharyngeal carcinoma in an era of intensity-modulated radiotherapy. BMC Cancer. 2015; 15: 977.

25. Zhang $\mathrm{B}, \mathrm{Mo} \mathrm{Z}, \mathrm{Du} \mathrm{W}$, et al. Intensity-modulated radiation therapy versus 2D-RT or 3D-CRT for the treatment of nasopharyngeal carcinoma: A systematic review and meta-analysis. Oral Oncol. 2015; 51(11): 1041-1046.

26. Lee AW, Tung SY, Ngan RK, et al. Factors contributing to the efficacy of concurrent-adjuvant chemotherapy for locoregionally advanced nasopharyngeal carcinoma: combined analyses of NPC-9901 and NPC-9902 Trials. Eur J Cancer. 2011; 47(5): 656-666.

27. Chen L, Hu CS, Chen XZ, et al. Concurrent chemoradiotherapy plus adjuvant chemotherapy versus concurrent chemoradiotherapy alone in patients with locoregionally advanced nasopharyngeal carcinoma: a phase 3 multicentre randomised controlled trial. Lancet Oncol. 2012; 13(2): 163-171.

28. Ribassin-Majed L, Marguet S, Lee AWM, et al. What Is the Best Treatment of Locally Advanced Nasopharyngeal Carcinoma? An Individual Patient Data Network Meta-Analysis. J Clin Oncol. 2017; 35(5): 498-505.

29. Wang XP, Li XH, Zhang L, et al. High level of serum apolipoprotein A-I is a favorable prognostic factor for overall survival in esophageal squamous cell carcinoma. BMC Cancer. 2016; 16: 516

30. Quan Q, Huang Y, Chen Q, et al. Impact of Serum Apolipoprotein A-I on Prognosis and Bevacizumab Efficacy in Patients with Metastatic Colorectal Cancer: a Propensity Score-Matched Analysis. Transl Oncol. 2017; 10(2): 288-294.

31. Qian H, Zhao X, Cao P, et al. Structure of the Human Lipid Exporter ABCA1. Cell. 2017; 169(7): 1228-1239.

32. Zamanian-Daryoush M, Lindner D, Tallant TC, et al. The cardioprotective protein apolipoprotein A1 promotes potent anti-tumorigenic effects. J Biol Chem. 2013; 288(29): 21237-21252.

33. Su F, Kozak KR, Imaizumi S, et al. Apolipoprotein A-I (apoA-I) and apoA-I mimetic peptides inhibit tumor development in a mouse model of ovarian cancer. Proc Natl Acad Sci U S A. 2010; 107(46): 19997-20002.

34. Sag D, Cekic C, Wu R, Linden J, Hedrick CC. The cholesterol transporter ABCG1 links cholesterol homeostasis and tumour immunity. Nat Commun. 2015; 6: 6354.

35. Yi ZF, Cho SG, Zhao H, et al. A novel peptide from human apolipoprotein(a) inhibits angiogenesis and tumor growth by targeting c-Src phosphorylation in VEGF-induced human umbilical endothelial cells. Int J Cancer. 2009; 124(4): 843-852.

36. Ren K, Lu YJ, Mo ZC, et al. ApoA-I/SR-BI modulates S1P/S1PR2-mediated inflammation through the PI3K/Akt signaling pathway in HUVECs. J Physiol Biochem. 2017; 73(2): 287-296.

37. Zheng $\mathrm{Y}$, Liu $\mathrm{Y}$, Jin $\mathrm{H}$, et al. Scavenger receptor $\mathrm{B} 1$ is a potential biomarker of human nasopharyngeal carcinoma and its growth is inhibited by HDL-mimetic nanoparticles. Theranostics. 2013; 3(7): 477-486.

38. Pang J, Chan DC, Hamilton SJ, et al. Effect of niacin on high-density lipoprotein apolipoprotein A-I kinetics in statin-treated patients with type 2 diabetes mellitus. Arterioscler Thromb Vasc Biol. 2014; 34(2): 427-432.

39. Kakuda $\mathrm{H}$, Matoba $\mathrm{M}$, Nakatoh $\mathrm{H}$, et al. Effects of change in high-density lipoprotein cholesterol by statin switching on glucose metabolism and renal function in hypercholesterolemia. J Clin Lipidol. 2015; 9(5): 709-715.

40. Park SM, Li T, Wu S, et al. Niacin intake and risk of skin cancer in US women and men. Int J Cancer. 2017; 140(9): 2023-2031.

41. Voorneveld PW, Reimers MS, Bastiaannet E, et al. Statin Use After Diagnosis of Colon Cancer and Patient Survival. Gastroenterology. 2017; 153(2): 470-479.

42. Vogel TJ, Goodman MT, Li AJ, Jeon CY. Statin treatment is associated with survival in a nationally representative population of elderly women with epithelial ovarian cancer. Gynecol Oncol. Gynecol Oncol. 2017; 146(2): 340-345. 